\title{
RESIDUAL SOLVABILITY OF AN EQUATION IN NILPOTENT GROUPS
}

\author{
PETER F. STEBE
}

\begin{abstract}
Let $G$ be a finitely generated nilpotent group. Let $S_{1}$ and $S_{2}$ be subgroups of $G$. Let $S_{1} S_{2}$ be the set of all products $g_{1} g_{2}$, where $g_{i}$ is an element of $S_{i}$. Let $g$ be an element of $G$. It is shown that either $g$ is an element of $S_{1} S_{2}$ or there is a normal subgroup $N$ of finite index in $G$ such that $g N$ does not meet $S_{1} S_{2}$. This result implies:

(a) There is an algorithm to determine whether or not $g$ is an element of $S_{1} S_{2}$.

(b) Given elements $a, b$, and $c$ of $G$, there is an algorithm to determine whether there exist integers $n$ and $m$ such that $a=b^{m} c^{n}$.

(c) Finitely generated nilpotent groups are subgroup separable (a result of K. Toh).

(d) Given elements $a$ and $b$ of $G$ and a subgroup $S$ of $G$, there is an algorithm to determine whether or not $a$ is an element of $S b S$.
\end{abstract}

Recall that every finitely generated nilpotent group $G$ is polycyclic, i.e. there exists a series $N_{i}$ such that $G=N_{0} \supset N_{1} \supset \cdots \supset N_{k}=1$, where $N_{i+1}$ is a normal subgroup of $N_{i}$ and the factor groups $N_{i} / N_{i+1}$ are cyclic. If $N_{i}$ and $M_{i}$ are any two such polycyclic series for $G$, it follows from inspection of a common refinement of the series that the number of infinite cyclic factor groups is independent of the series selected. The number of infinite cyclic factor groups for any polycyclic series of $G$ is called the torsion free rank of $G$. Clearly, if $C$ is an infinite cyclic normal subgroup of $G$, the torsion free rank of $G / C$ is one less than that of $G$. Recall also that if $G$ is an infinite finitely generated nilpotent group, there is an element of infinite order in the center of $G$. These remarks allow us to use induction on the torsion free rank of $g$, as in the monograph by G. Baumslag [1]. This reference is the general reference for theorems on nilpotent groups.

TheOREM. Let $G$ be a finitely generated nilpotent group. Let $S_{1}$ and $S_{2}$ be subgroups of $G$. If $g$ is an element of $G$, then either $g$ is an element of $S_{1} S_{2}$ or there is a normal subgroup $N$ of finite index in $G$ such that $g N$ does not meet $S_{1} S_{2}$.

Proof. Let $Z$ be the center of $G$. Note that if $z$ is an element of $S_{1} S_{2}$ and an element of $Z$, then the cyclic subgroup generated by $z$ is contained in $S_{1} S_{2}$. Let $z=s_{1} s_{2}$, where $s_{i}$ is contained in $S_{i}$. We have $s^{-1} z=s_{2}$, so that $\left(s_{1}^{-1} z\right)^{k}=s_{2}^{k}$ for all integers $k$. Since $z$ is central, $s_{1}^{-k} z^{k}=s_{2}^{k}$, and so $z^{k}$ $=s_{1}^{k} s_{2}^{k}$.

Received by the editors January 27, 1975.

AMS (MOS) subject classifications (1970). Primary 20E25.

Key words and phrases. Residual property, nilpotent group.

(1) American Mathematical Society 1976 
The proof of the theorem is by induction on the torsion free rank of $G$. If $G$ has torsion free rank zero, $G$ is finite and the result follows with $N$ the identity subgroup. Assume $G$ has positive torsion free rank and that the result is valid for groups of lesser torsion free rank. Let $z$ be an element of infinite order in $Z$. If, for a given nonzero integer $k, g$ is not contained in $S_{1} S_{2}\left\langle z^{k}\right\rangle$, the result follows, for $\left\langle z^{k}\right\rangle$ is normal in $G$ and $G /\left\langle z^{k}\right\rangle$ has lesser torsion free rank than $G$. (Note that $\left\langle z^{k}\right\rangle$ is the subgroup generated by $z^{k}$.) We can thus assume that $g$ is an element of $S_{1} S_{2}\left\langle z^{k}\right\rangle$ for all nonzero $k$. It will follow that this implies that $g$ is an element of $S_{1} S_{2}$.

Since $g \in S_{1} S_{2}\langle z\rangle$, there are elements $s_{1}$ and $s_{2}$ of $S_{1}$ and $S_{2}$, respectively, and an integer $i$ such that $g=s_{1} s_{2} z^{i}$. Since $g \in S_{1} S_{2}\left\langle z^{1+i^{2}}\right\rangle$, there are elements $c_{1}$ and $c_{2}$ of $S_{1}$ and $S_{2}$ and an integer $u$ such that $g=c_{1} c_{2} z^{u\left(1+i^{2}\right)}$. If either $i$ or $u$ is zero, we are finished. If $i$ and $u$ are not zero, $v=u\left(1+i^{2}\right)-i$ is not zero, since $i$ and $1+i^{2}$ are relatively prime. Equating the two expressions for $g$ and recalling that $z$ is central, $c_{1}^{-1} s_{1} s_{2} c_{2}^{-1}=z^{u\left(1+i^{2}\right)-i}$. Thus $z^{v}$ is a central element of $S_{1} S_{2}$. By the lemma at the start of the proof, the entire cyclic subgroup generated by $z^{v}$ is in $S_{1} S_{2}$. Since $v$ is not zero, it follows by assumption that

$$
g \in S_{1} S_{2}\left\langle z^{v}\right\rangle=S_{1}\left\langle z^{v}\right\rangle S_{2} \subset S_{1} S_{1} S_{2} S_{2}=S_{1} S_{2}
$$

REMARKS. The result (a) follows from standard methodsof logic and the restatement of the result is as follows: Let $g$ be an element of $G$. Either $G$ is in the complex $S_{1} S_{2}$ or there is a homomorphism $\theta$ of $G$ onto a finite group such that $g \theta \notin S_{1} \theta S_{2} \theta$.

The result (b) is the case of $S_{1}$ and $S_{2}$ cyclic. This case has been considered in the study of other groups [2].

Subgroup separability is the case of one of the subgroups being the identity group. This result was obtained by K. H. Toh [3].

The problem solved in (d) is called the double coset problem. We replace the formula $a \in S b S$ by the equivalent formula $a b^{-1} \in S b S b^{-1}$ and set $S_{1}=S, S_{2}=b S b^{-1}$ in the theorem.

\section{REFERENCES}

1. G. Baumslag, Lecture notes on nilpotent groups, Regional Conference Series in Math., no. 2, Amer. Math. Soc., Providence, R.I., 1971. MR 44 \#315.

2. P. F. Stebe, Conjugacy separability of certain free products with amalgamation, Trans. Amer. Math. Soc. 156 (1971), 119-129. MR 43 \#360.

3. K. Toh, Problems concerning residual finiteness in nilpotent groups, Dept. of Math., Univ. of Malaya, Kuala Lumpur, Malaysia.

Department of Mathematics, City College, New York, New York 10031 